\title{
Validação de uma metodologia analítica para determinação de cálcio em produtos cárneos
}

\author{
Validation of a analytical methodology for calcium determination in meat products
}

\author{
Rafael BURIN ${ }^{1}$, Vivian Maria BURIN ${ }^{1}$, Patrícia TAHA ${ }^{1}$, Marilde Terezinha BORDIGNON-LUIZ ${ }^{1 *}$
}

\section{Resumo}

O objetivo deste trabalho consiste em validar uma otimização para uma metodologia tradicional de determinação de cálcio em produtos cárneos. No Brasil, esta metodologia é descrita pelo Ministério da Agricultura Pecuária e Abastecimento e utiliza como elemento filtrante, em uma das etapas de filtração, fibras médias de amianto, um material tóxico. Na metodologia proposta neste trabalho, estas fibras foram substituídas por papel filtro quantitativo. Os testes realizados foram: linearidade, sensibilidade, limite de detecção e de quantificação, precisão, exatidão, especificidade, robustez e incerteza da medição. A metodologia otimizada proposta neste trabalho mostrou-se sensível, precisa, exata, específica e robusta. Pode-se considerar esta metodologia adequada para determinar a concentração de cálcio em produtos cárneos. Palavras-chave: validação de metodologia; cálcio; produtos cárneos.

\begin{abstract}
The aim of this study was to validate an optimization of a traditional methodology of calcium determination in meat products. In Brazil, the method of calcium determination described by the Ministério da Agricultura Pecuária e Abastecimento uses asbesto fibers in a stage of filtration, which is toxic. In the methodology proposed in this work, we replaced these asbestos fibers with quantitative filter paper. To evaluate the analytical methodology proposed, we considered: linearity, sensitivity, detection, quantification limits, precision, accuracy, specificity, robustness, and inaccuracy of measurements. The optimized analytical method proposed in this work proved sensible, accurate, exact, robust, and linear. Considering the determination of the calcium content in meat products was considered suitable.

Keywords: validation methods; calcium; meat products.
\end{abstract}

\section{Introdução}

Em produtos cárneos, a concentração de cálcio depende do tipo de embutido e da quantidade de carne mecanicamente separada (CMS) utilizada para a sua fabricação. A legislação brasileira limita o uso de CMS nestes produtos (SOUZA et al., 2003).

A metodologia analítica para determinação de cálcio em produtos cárneos recomendada pelo Ministério da Agricultura Pecuária e Abastecimento (MAPA), descrita em Brasil (1999), descreve em uma das etapas de filtração, a utilização de cadinho de Gooch e, como elemento filtrante, fibras médias de amianto. Estas são material tóxico que, segundo D’acri (2003), pode causar asbestose pulmonar, câncer de pulmão, mesoteliomas e doenças pleurais benignas, como placas pleurais e limitações crônicas de fluxo aéreo.

É fundamental que os laboratórios disponham de meios e critérios objetivos para demonstrar, por meio da validação, que as metodologias de ensaio que executam conduzem a resultados confiáveis e adequados à qualidade pretendida (RIBANI, 2004). Se uma metodologia for modificada para atender aos requisitos específicos ou um método novo for desenvolvido, o laboratório deve assegurar que as características de desempenho do método atendam aos requisitos para as operações analíticas pretendidas (INMETRO, 2003).

Os termos 'validação' e 'garantia da qualidade' são extensamente usados. Segundo a Associação Brasileira de Normas Técni- cas (NBR ISO/IEC 17025), validar um método é confirmar - por exame e fornecimento de evidência objetiva - que os requisitos específicos para um determinado uso pretendido são atendidos. Validação de metodologias analíticas é o primeiro passo para a garantia da qualidade em um laboratório. A garantia da qualidade analítica é o conjunto completo das medidas que um laboratório deve assegurar, a fim de conseguir dados de alta qualidade. Além do uso da validação e/ou das metodologias padronizadas, há outras medidas que devem ser seguidas, como: procedimentos eficazes de controle da qualidade interno (uso de materiais de referência, calibração de equipamentos, controle de documentos, etc.); participação em testes de proficiência, e acreditação a um organismo internacional, normalmente NBR ISO/IEC 17025 (TAVERNIERS; LOOSE; BOCKSTAELE, 2004). Os critérios de seleção para o procedimento apropriado de validação dependerão das condições do laboratório, do método utilizado, do tipo de amostra, dos componentes do mensurando, da calibração dos equipamentos e da disponibilidade de materiais de referência certificados.

\section{Material e métodos}

\subsection{Material}

Para a determinação de cálcio, foi utilizada uma amostra de salsicha, adquirida em mercado local. O padrão utilizado foi

Recebido para publicação em 15/10/2007

Aceito para publicação em 27/5/2008 (002917)

${ }^{1}$ Departamento de Ciência e Tecnologia de Alimentos, Universidade Federal Santa Catarina - UFSC, CEP 88034-001, Florianópolis - SC, Brasil, E-mail: bordign@cca.ufsc.br

${ }^{*}$ A quem a correspondência deve ser enviada 
Material De Referência Certificado (MRC), carbonato de cálcio (FLUKA) pureza de $99,70 \%$ e incerteza expandida de $\pm 0,13 \%$, com nível de confiança de $95 \%$. A concentração da solução padrão foi de 1,0013 mg de cálcio/mL. Os demais reagentes utilizados foram de qualidade p.a. e os equipamentos e vidrarias foram calibrados segundo a Rede Brasileira de Calibração (RBC).

\subsection{Métodos}

\section{Procedimento para análise}

A determinação de cálcio para produtos cárneos foi realizada em amostra de salsicha, segundo metodologia proposta por Brasil (1999) com modificações. Uma das etapas de filtração deste método utiliza cadinho de Gooch e, como elemento filtrante, fibras médias de amianto, que foram substituídas por papel filtro quantitativo.

A amostra (5 g) foi pesada (Balança analítica Shimadzu AY-220, Japão) em cadinho, carbonizada em placa aquecedora (Tecnal, Brasil), incinerada em forno mufla (Quimis, Brasil) $\left(550 \pm 5^{\circ} \mathrm{C}\right)$ e esfriada a temperatura ambiente. Em seguida, foi adicionado ácido clorídrico concentrado $(5,0 \mathrm{~mL})$ e este foi evaporado em chapa aquecedora $\left(100^{\circ} \mathrm{C}\right)$. Nova adição de ácido clorídrico concentrado $(2,0 \mathrm{~mL})$ foi realizada e o cadinho foi coberto com vidro relógio e aquecido em placa aquecedora $\left(100^{\circ} \mathrm{C}\right)$ durante 5 minutos. Após resfriamento da amostra, o cadinho foi lavado com água deionizada, seguido de uma filtração em papel filtro qualitativo. $\mathrm{O}$ filtrado foi recolhido em um balão volumétrico de $100 \mathrm{~mL}$. Esse material foi denominado como "solução estoque".

Foram transferidos $50,0 \mathrm{~mL}$ da solução estoque para um erlenmeyer de $250 \mathrm{~mL}$ e adicionadas 3 gotas de solução alcoólica de vermelho de metila $(0,1 \% \mathrm{~m} / \mathrm{v})$ e $50 \mathrm{~mL}$ de água deionizada. A solução foi neutralizada com a utilização de hidróxido de amônio $(1+1) \mathrm{v} / \mathrm{v}$, ajustado o $\mathrm{pH}(5,5)$ com $\mathrm{HCl}(1+9) \mathrm{v} / \mathrm{v}$, e mantida em ebulição (1 minuto) em placa aquecedora. Posteriormente, foi adicionado oxalato de amônio $4,2 \% \mathrm{~m} / \mathrm{v}(10,0 \mathrm{~mL})$. No repouso (12 horas), foi observada a formação do precipitado de oxalato de cálcio por meio de uma reação química entre o cálcio e o oxalato. Após este período, o precipitado obtido foi filtrado em papel filtro quantitativo (Vetec - cód. 050150). Nesta etapa da metodologia proposta por Brasil (1999), é utilizado o amianto como elemento filtrante. O papel filtro foi lavado com hidróxido de amônio $2 \% \mathrm{v} / \mathrm{v}(50 \mathrm{~mL})$, para retirar o excesso de oxalato. Os filtrados foram descartados. O papel filtro contendo o precipitado de oxalato de cálcio foi transferido para um funil de vidro e lavado, cuidadosamente, com ácido sulfúrico $(5+125) \mathrm{v} / \mathrm{v}$ $(130 \mathrm{~mL})$. O ácido oxálico formado foi titulado com permanganato de potássio $0,01 \mathrm{M}$, a $75^{\circ} \mathrm{C}$ (banho termostatizado (Tecnal, Brasil). O teor de cálcio foi calculado, considerando-se que cada mililitro da solução padronizada de permanganato de potássio gasto na titulação corresponde a 1,0 mg de cálcio.

\subsection{Validação da metodologia proposta}

\section{Faixa de trabalho e linearidade}

A faixa de trabalho e a linearidade foram determinadas de acordo com especificações do Inmetro (2003). Para o cálculo da faixa de trabalho, foi construída uma curva utilizandose solução padrão de cálcio com seis níveis de calibração $(1,3,5,10,15$ e $20 \mathrm{mg}$ de cálcio). O número de replicatas para cada nível de calibração foi sete.

A linearidade do método foi determinada a partir da curva da faixa de trabalho e calculada a partir da equação da regressão linear (Equação 1) (EUROPEAN AGENCY FOR THE EVALUATION OF MEDICINAL PRODUCTS, 2007).

$\mathrm{y}=\mathrm{ax}+\mathrm{b}$

sendo:

$\mathrm{y}=$ resposta medida; $\mathrm{x}=$ concentração; $\mathrm{a}=$ inclinação da curva de calibração (sensibilidade); b = interseção com o eixo y

Sensibilidade, limite de detecção $(L D) e$

limite de quantificação (LQ)

A sensibilidade foi determinada a partir da inclinação da curva na faixa de trabalho (TAVERNIERS; LOOSE; BOCKSTAELE, 2004) e calculada de acordo com a Equação 2.

$S=\frac{d x}{d y}$

sendo:

$\mathrm{S}=$ sensibilidade; $\mathrm{dx}=$ variação da resposta; $\mathrm{e} \mathrm{dy}$ = variação da concentração

O LD foi determinado pela análise do branco (todos os reagentes utilizados na análise sem a adição do analito) com 10 repetições, sendo calculados a média e o desvio padrão. $\mathrm{O}$ LD foi calculado pela soma das médias dos brancos mais três vezes o desvio padrão dividido pela sensibilidade do método (Equação 3) (FEINBERG; RAGUĖNÈS, 1998).

$L D=\frac{\bar{x}+3 . s}{S}$

sendo:

$\mathrm{x}=$ média dos valores dos brancos da amostra; $\mathrm{s}=$ desvio padrão dos brancos da amostra; e $\mathrm{S}=$ sensibilidade

Para a determinação do Limite de Quantificação (LQ) (Equação 4), foram analisados 10 brancos e calculados a média e o desvio padrão (FEINBERG; RAGUÈNÈS, 1998).

$L Q=\frac{\bar{x}+10 . s}{S}$

sendo:

$\mathrm{x}=$ média dos valores dos brancos; $\mathrm{s}=$ desvio padrão dos brancos; e $\mathrm{S}=$ sensibilidade do método

\section{Precisão}

A precisão foi avaliada a partir da repetitividade dos resultados, utilizando-se a curva da faixa de trabalho (MOHAMED et al., 2005). A precisão da metodologia foi expressa em termos de desvio padrão relativo (NEVADO et al., 2005). 


\section{Exatidão}

Para a avaliação da exatidão da metodologia, foi calculado o erro relativo de acordo com a Equação 5. O índice $Z$ foi calculado segundo a Equação 6. A avaliação dos resultados do índice $Z$ foi realizada de acordo com a seguinte escala de pontuação: $|Z| \leq 2$ = satisfatório; $2<|Z| \leq 3=$ questionável; $|Z|>3=$ insatisfatório (INMETRO, 2003).

A recuperação do analito também foi avaliada em amostras fortificadas: amostras de salsichas foram adicionadas de material de referência certificado em 3 diferentes concentrações (2, 5 e $10 \mathrm{mg}$ cálcio). As análises foram realizadas em triplicata (GARBERLOTTI; TORRES; MARSIGLIA, 2003).

$E R=\frac{X_{l a b}-X_{v}}{X_{v}} \cdot 100$

sendo:

$\mathrm{X}_{\mathrm{lab}}=$ média dos valores obtidos na análise da solução padrão; $\mathrm{X}_{\mathrm{v}}$ = valor teórico aceito como verdadeiro Material de Referência Certificado (MRC)

$Z=\frac{\left|X_{l a b}-X_{v}\right|}{s}$

sendo:

$\mathrm{X}_{\mathrm{lab}}=$ média dos valores obtidos experimentalmente; $\mathrm{X}_{\mathrm{v}}=$ valor teórico aceito como verdadeiro (MRC); $s=$ unidade de desvio (incerteza do MRC).

\section{Especificidade}

A análise da especificidade da metodologia foi realizada com solução padrão de cálcio (MRC) adicionada de impurezas (PLATZER; WHITE, 2006). Foram pipetadas alíquotas de 5,0 $\mathrm{mL}$ do padrão de cálcio em 10 cadinhos de porcelana: 5 cadinhos foram contaminados com $2,0 \mathrm{~mL}$ de uma solução de magnésio ( $6 \mathrm{mg}$ de $\mathrm{Mg}^{2+} / \mathrm{mL}$ ) e nos outros 5 cadinhos foram adicionados $0,5 \mathrm{~mL}$ de solução de sulfato de sódio $(33,5 \mathrm{mg}$ de $\mathrm{SO}_{4}^{-2} / \mathrm{mL}$ ), realizando-se todo o procedimento analítico.
Robustez

Para avaliar a robustez da metodologia, foi utilizado o teste proposto por Youden e Steiner (TAN; XU; ZHENG, 1995) conforme a Tabela 1. Este teste permite avaliar a robustez e também ordenar a influência de cada uma das variações nos resultados finais, indicando qual o tipo de influência que cada variação exerce. Para determinar a variação de um fator, foram encontrados os quatro valores correspondentes às letras maiúsculas e minúsculas, comparando-se as médias dos dois grupos. Para calcular as variações de A para a, foi usada a Equação 7.

$\frac{A}{a}=\frac{(s+t+u+v)}{4}-\frac{(x+y+w+z)}{4}$

A Tabela 1 mostra quais os fatores que foram modificados para a determinação da robustez. Foram denominados os fatores nominais por letras maiúsculas de $\mathrm{A}$ a $\mathrm{G}$ e as variações, por letras minúsculas correspondentes. Estes fatores e suas variações foram escolhidos pelo analista de acordo com testes preliminares.

\section{Incerteza de medição}

A incerteza da medição foi avaliada de acordo com Ellison, Rosslein e Willians (2002), em quatro etapas: 1)etapa foi a especificação do mensurando; 2) identificação das fontes de incerteza; 3) quantificação dos componentes de incerteza; e 4) cálculo da incerteza combinada.

\subsection{Análise estatística}

A análise estatística foi realizada utilizando-se o software STATISTICA * versão 6.0 (2001) com a aplicação do teste ANOVA. Para realização da curva de regressão linear na faixa de trabalho, média, desvio padrão e variância, foi utilizado o programa Excel 2000 for Windows.

\section{Resultados e discussão}

\subsection{Faixa de trabalho e linearidade}

Os resultados da faixa de trabalho estão indicados na Tabela 2 e na Figura 1. O método demonstrou uma boa lineari-

Tabela 1. Modelo experimental para teste de robustez.

\begin{tabular}{|c|c|c|c|c|c|c|c|c|c|c|c|}
\hline \multirow[t]{2}{*}{ Variáveis } & \multicolumn{8}{|c|}{ Experimentos } & \multicolumn{3}{|c|}{ Variações dos fatores } \\
\hline & 1 & 2 & 3 & 4 & 5 & 6 & 7 & 8 & Efeito & Nominal & Variação \\
\hline $\mathrm{V} 1$ & $\mathrm{~A}$ & A & A & $\mathrm{A}$ & $\mathrm{a}$ & $\mathrm{a}$ & $\mathrm{a}$ & $\mathrm{A}$ & $\mathrm{A} / \mathrm{a}$ & $75^{\circ} \mathrm{C}$ & $90^{\circ} \mathrm{C}$ \\
\hline $\mathrm{V} 2$ & $\mathrm{~B}$ & B & $\mathrm{b}$ & $\mathrm{b}$ & B & B & $\mathrm{b}$ & $\mathrm{b}$ & $\mathrm{B} / \mathrm{b}$ & $130 \mathrm{~mL}$ & $100 \mathrm{~mL}$ \\
\hline $\mathrm{V} 3$ & $\mathrm{C}$ & $\mathrm{c}$ & $\mathrm{C}$ & c & C & $\mathrm{c}$ & $\mathrm{C}$ & $\mathrm{c}$ & $\mathrm{C} / \mathrm{c}$ & $2 \% \mathrm{v} / \mathrm{v}$ & $4 \% \mathrm{v} / \mathrm{v}$ \\
\hline $\mathrm{V} 4$ & $\mathrm{D}$ & $\mathrm{D}$ & $\mathrm{d}$ & $\mathrm{d}$ & $\mathrm{d}$ & $\mathrm{d}$ & $\mathrm{D}$ & $\mathrm{D}$ & $\mathrm{D} / \mathrm{d}$ & 12 horas & 2 horas \\
\hline V5 & $\mathrm{E}$ & $\mathrm{e}$ & $\mathrm{E}$ & $\mathrm{e}$ & $\mathrm{e}$ & $\mathrm{E}$ & $\mathrm{e}$ & $\mathrm{E}$ & $\mathrm{E} / \mathrm{e}$ & $\begin{array}{c}\text { Após } 1 \text { minuto de } \\
\text { ebulição }\end{array}$ & $\begin{array}{l}\text { Início da } \\
\text { ebulição }\end{array}$ \\
\hline V6 & $\mathrm{F}$ & $\mathrm{f}$ & $\mathrm{f}$ & $\mathrm{F}$ & $\mathrm{F}$ & $\mathrm{f}$ & $\mathrm{f}$ & $\mathrm{F}$ & $\mathrm{F} / \mathrm{f}$ & 5,5 & 4,0 \\
\hline V7 & G & $\mathrm{g}$ & $\mathrm{g}$ & G & $\mathrm{g}$ & G & G & $\mathrm{g}$ & G/g & $\begin{array}{c}\text { Leve } \\
\text { agitação }\end{array}$ & $\begin{array}{c}\text { Forte } \\
\text { agitação }\end{array}$ \\
\hline Resultados & $\mathrm{s}$ & $\mathrm{t}$ & $\mathrm{u}$ & $\mathrm{v}$ & $\mathrm{x}$ & $\mathrm{y}$ & $\mathrm{u}$ & $\mathrm{V}$ & $\mathrm{Z}$ & Nominal & Variação \\
\hline
\end{tabular}

V1 = temperatura de titulação; V2 = volume da solução de ácido sulfúrico $(5+125)$ v/v; V3 = concentração da solução de hidróxido de amônio; V4 = tempo de precipitação; V5 = tempo de adição da solução de oxalato de amônio $4,2 \% \mathrm{~m} / \mathrm{v}$; V6 = pH de precipitação; e V7 = agitação durante a titulação. 
dade, $\mathrm{R}^{2}=0,9999$, na medida em que, segundo a FDA (2007), o critério mínimo aceitável para o coeficiente de correlação linear deve ser maior ou igual a 0,995 . A equação da reta obtida foi $y=1,0106 x-0,0527$.

Os resultados da linearidade da metodologia na faixa de trabalho não dependem apenas da sensibilidade do equipamento utilizado, pois outros aspectos devem ser observados: a complexidade do método, os reagentes utilizados, a extração do analito, as condições do equipamento e do meio ambiente, além da habilidade do analista. No desenvolvimento e validação de métodos para determinar traços de ftalatos (ésteres de ácido ftálico) em vegetais, Sablayrolles et al. (2005) utilizaram o método da cromatografia gasosa com espectroscopia de massa, obtendo coeficientes de correlação linear na faixa de 0,7247 a 0,9742 para baixas concentrações e 0,8840 a 0,9852 para concentrações altas. Gaberlotti, Marsiglia e Torres (2003) validaram um método enzimático gravimétrico para determinação de fibras dietéticas em alimentos, sendo o coeficiente de correlação linear igual a 0,9999 - indicativo de uma boa linearidade do método - e a curva de calibração construída com 3 níveis de concentração. Pinho et al. (2000), na quantificação de antioxidantes sintéticos em patês de fígado por cromatografia líquida de alta eficiência (CLAE), utilizando-se uma curva de calibração com 5 níveis de concentração, obtiveram uma linearidade superior a 0,99. Nestas pesquisas, evidenciou-se uma melhor linearidade para o método gravimétrico quando comparado ao método por cromatografia.

Tabela 2. Resultados corrigidos da faixa de trabalho, com o desvio padrão e o Coeficiente de Variação (CV)

\begin{tabular}{cccc}
\hline $\begin{array}{c}\text { Massa de cálcio } \\
\text { esperada* }(\mathrm{mg})\end{array}$ & $\begin{array}{c}\text { Massa de cálcio } \\
\text { obtida }(\mathrm{mg})\end{array}$ & $\begin{array}{c}\text { Repetitividade** } \\
\text { (desvio padrão) }\end{array}$ & CV\% \\
\hline 0,993 & 1,001 & 0,028 & 2,75 \\
2,997 & 2,891 & $\pm 0,046$ & 1,60 \\
4,995 & 4,863 & $\pm 0,028$ & 0,56 \\
9,990 & 10,160 & $\pm 0,039$ & 0,38 \\
14,991 & 15,141 & $\pm 0,042$ & 0,28 \\
19,980 & 20,088 & $\pm 0,091$ & 0,45 \\
\hline
\end{tabular}

${ }^{*} \mathrm{O}$ valor teórico é o valor corrigido da quantidade de cálcio na alíquota; $\mathrm{e}^{* *}$ para cada nível de concentração $\mathrm{n}=7$.

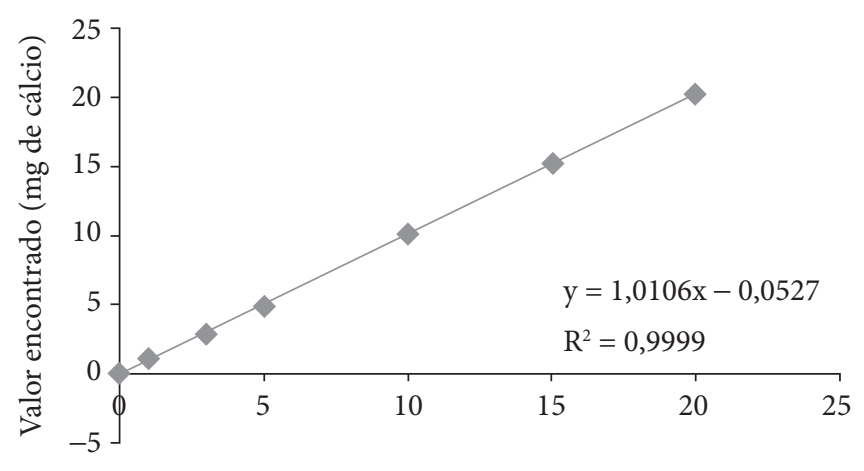

Valor nominal (mg de cálcio)

Figura 1. Linearidade e faixa de trabalho para o método de determinação de cálcio.

\subsection{Sensibilidade, limite de detecção e limite de quantificação}

A sensibilidade da metodologia calculada utilizando-se a curva de regressão linear foi de 1,0057 mg de cálcio.

A média $(n=10)$ dos valores do branco (reagentes utilizados na análise sem a adição do analito) foi de $0,40 \mathrm{~mL}$ de solução de permanganato de potássio, apresentando um desvio padrão de $\pm 0,04$.

O limite de detecção observado para o método foi de 0,52 mg de cálcio/5 g de amostra ou $11 \mathrm{mg}$ de cálcio/100 g de amostra. O limite de quantificação observado foi de $0,80 \mathrm{mg}$ de cálcio/5 g de amostra ou $16 \mathrm{mg}$ de cálcio/100 g de amostra.

\subsection{Precisão}

A precisão na faixa de trabalho é apresentada na forma de repetitividade (Tabela 2) sendo avaliado o desvio padrão dos resultados obtidos. Ainda nesta tabela pode-se notar que os resultados dos coeficientes de variação (\% CV) foram aceitáveis, pois não foi observado valor superior a 5\%, que é o limite máximo admitido por Brasil (2003). O FDA (2007) estabelece como critério de aceitação para o coeficiente de variação valor menor ou igual a $2 \%$, sendo que somente o primeiro nível de concentração $(1,00 \mathrm{~mL})$ da curva de calibração ficou acima do limite.

\subsection{Exatidão}

Os resultados da recuperação do analito em amostras fortificadas com solução padrão de cálcio estão apresentados na Tabela 3. Foi obtida uma boa recuperação, pois de acordo com a FDA (2007) os limites para recuperação do analito são de 80 a $120 \%$.

A média dos resultados do índice $\mathrm{Z}$ e o erro relativo do método estão descritos na Tabela 4 . O erro relativo do método apresentou uma variação menor que $5 \%$ para todos os níveis de concentrações. Os resultados do índice $Z$ foram satisfatórios (menor que 2) para todas as concentrações da solução padrão de cálcio.

A forma mais utilizada pelos pesquisadores para avaliar a exatidão de método é por meio da porcentagem de recuperação do analito. Garda, Macedo e Fourlong (2004) determinaram tricotecenos em cerveja pela análise de duas toxinas (DON e T-2). Utilizando-se a cromatografia gasosa, obtiveram a re-

Tabela 3. Médias $(n=7)$ dos resultados de recuperação do analito em amostras fortificadas.

\begin{tabular}{cccc}
\hline Amostra $(\mathrm{g})$ & $\begin{array}{c}\text { mg de cálcio } \\
\text { teórico }\end{array}$ & $\begin{array}{c}\text { mg de cálcio } \\
\text { detectado }\end{array}$ & $\begin{array}{c}\text { Recuperação do } \\
\text { analito (\%) }\end{array}$ \\
\hline 6,038 & 0,000 & 6,532 & 0,00 \\
6,051 & 2,997 & 9,635 & 101,01 \\
6,046 & 4,995 & 11,546 & 100,17 \\
6,040 & 9,990 & 16,492 & 99,80 \\
\hline
\end{tabular}

Cálcio teórico = cálcio adicionado. 
Tabela 4. Exatidão do método avaliada pelo erro relativo (\%) e índice $\mathrm{Z}$ (z score) a partir dos resultados obtidos na faixa de trabalho.

\begin{tabular}{cccc}
\hline $\begin{array}{c}\text { Massa de cálcio } \\
\text { esperada }^{*}(\mathrm{mg})\end{array}$ & $\begin{array}{c}\text { Massa de cálcio } \\
\text { obtida }(\mathrm{mg})\end{array}$ & Índice $|\mathrm{Z}|$ & Erro relativo (\%) \\
\hline 0,993 & 1,001 & 0,06 & 0,78 \\
2,997 & 2,891 & 0,81 & $-3,52$ \\
4,995 & 4,863 & 1,01 & $-2,64$ \\
9,990 & 10,160 & 1,31 & 1,71 \\
14,991 & 15,141 & 1,16 & 1,00 \\
19,980 & 20,088 & 0,83 & 0,54 \\
\hline
\end{tabular}

cuperação da toxina DON de 81,5 a $84,6 \%$ e da toxina T-2 de 101,0 a 105,0\%. De acordo com Garberlotti, Torres e Marsiglia w(2003), na validação do método enzimático gravimétrico para determinação de fibras dietéticas em alimentos, os percentuais de recuperação do padrão foram de 101, 108 e 110\%, admitindose uma escala de tolerância de 70 a $120 \%$.

\subsection{Especificidade}

Os resultados da contaminação com solução de magnésio e solução de sulfato estão descritos na Tabela 5. As contaminações da solução padrão não interferiram na recuperação do analito $\left(\mathrm{Ca}^{2+}\right)$, indicando que o oxalato tem preferência pelo cálcio no pH utilizado para precipitação. Tal ocorrência era esperada, uma vez que o produto de solubilidade do oxalato de magnésio é cerca de 10 mil vezes maior que o do oxalato de cálcio. Este fato é muito importante na determinação de cálcio por métodos titrimétricos, os quais freqüentemente sofrem interferência do magnésio.

\subsection{Robustez}

Os resultados dos ensaios das combinações das variações estão descritos na Tabela 6, que indica também os resultados das recuperações.

A Figura 2 apresenta graficamente as influências das variações no método. Nota-se que a variação na concentração da solução de hidróxido de amônio - solução utilizada para retirar o excesso de oxalato do papel filtro - foi a que apresentou a menor porcentagem de recuperação do analito, com 95,31\%. Observa-se que a variação da concentração utilizada foi o dobro da recomendada pelo método proposto, o que pode ter influenciado para a baixa recuperação obtida. Esta ocorrência pode ser justificada, pois o cálcio é deslocado do oxalato para formar hidróxido de cálcio, o qual apresenta solubilidade aproximadamente mil vezes maior que o oxalato de cálcio, sendo este eliminado na filtração.

O resultado da avaliação de cada uma das variáveis foi observado pelos efeitos $\mathrm{A} / \mathrm{a}=0,0188 ; \mathrm{B} / \mathrm{b}=0,0033 ; \mathrm{C} / \mathrm{c}=0,0633$; $\mathrm{D} / \mathrm{d}=0,0280 ; \mathrm{E} / \mathrm{e}=0,0086 ; \mathrm{F} / \mathrm{f}=0,0132$ e G/g = 0,0045. Como pode ser observado na Figura 2, o maior efeito foi o $\mathrm{C} / \mathrm{c}=0,0633$, que teve a maior influência no resultado.

\subsection{Incerteza da medição}

Esta fase compreendeu quatro etapas: 1) especificação do mensurando; 2) identificação das fontes de incerteza;3) quanti-
Tabela 5. Médias $(n=5)$ de recuperação do cálcio com interferência de íons $\mathrm{Mg}^{+2} \mathrm{e} \mathrm{SO}_{4}^{-2}$.

\begin{tabular}{|c|c|c|c|c|c|}
\hline $\begin{array}{l}\text { Padrão de } \\
\text { cálcio* } \\
(\mathrm{mL})\end{array}$ & $\begin{array}{l}\text { Padrão de } \\
\text { magnésio } \\
(\mathrm{mL})\end{array}$ & $\begin{array}{l}\text { Padrão de } \\
\text { sulfato }^{* * *} \\
(\mathrm{~mL})\end{array}$ & $\begin{array}{c}\mathrm{Ca}++ \\
\text { detectado } \\
(\mathrm{mg})\end{array}$ & $\begin{array}{c}\text { Recuperação } \\
(\%)\end{array}$ & $\begin{array}{c}\text { Erro } \\
\text { relativo } \\
(\%)\end{array}$ \\
\hline 5,0 & 2,0 & 0,0 & 5,018 & 100,50 & 0,47 \\
\hline 5,0 & 0,0 & 0,5 & 4,968 & 99,45 & 0,54 \\
\hline
\end{tabular}

Tabela 6. Resultados das recuperações dos fatores nominais e das variações.

\begin{tabular}{ccc}
\hline Variáveis* $^{*}$ & Níveis & Recuperação (\%) \\
\hline V1 & $75^{\circ} \mathrm{C}(\mathrm{A})$ & 100,67 \\
& $90{ }^{\circ} \mathrm{C}(\mathrm{a})$ & 99,31 \\
$\mathrm{~V} 2$ & $130 \mathrm{~mL}(\mathrm{~B})$ & 100,67 \\
& $100 \mathrm{~mL}(\mathrm{~b})$ & 99,51 \\
V3 & $2 \% \mathrm{v} / \mathrm{v}(\mathrm{C})$ & 100,67 \\
& $4 \% \mathrm{v} / \mathrm{v}(\mathrm{c})$ & 95,31 \\
V4 & 12 horas (D) & 100,67 \\
& 2 horas (d) & 98,74 \\
V5 & Após 1' de ebulição (E) & 100,67 \\
& No início da ebulição (e) & 99,90 \\
V6 & $5,5(\mathrm{~F})$ & 100,67 \\
& $4,0(\mathrm{f})$ & 99,76 \\
V7 & Leve (G) & 100,67 \\
& Forte (g) & 100,34 \\
\hline
\end{tabular}

${ }^{*}$ As variações intencionais no método foram: $\mathrm{V} 1=$ temperatura de titulação; $\mathrm{V} 2=$ volume da solução de ácido sulfúrico $(5+125)$ v/v; V3 = concentração da solução de hidróxido de amônio; V4 = tempo de precipitação; V5 = tempo de adição da solução de oxalato de amônio 4,2\% m/v; V6 = pH de precipitação; e V7 = agitação durante a titulação.

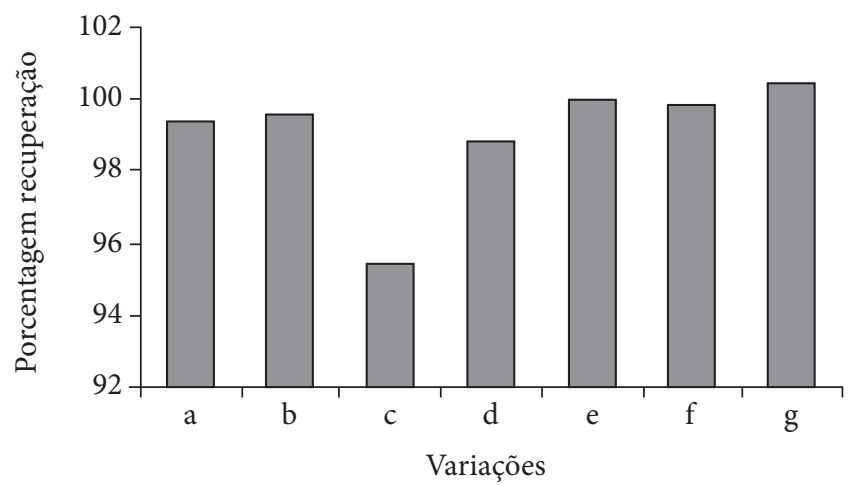

Figura 2. Porcentagem de recuperação do analito para cada variação, $\mathrm{a}=$ temperatura de titulação; $\mathrm{b}=$ volume solução de ácido sulfúrico; $\mathrm{c}=$ concentração da solução de hidróxido de amônio; $\mathrm{d}=$ tempo de precipitação; e = adição da solução de oxalato de amônio; $f=p H$ de precipitação; e g = agitação durante a titulação.

ficação dos componentes de incerteza, e 4) cálculo da incerteza combinada. Na primeira etapa, o mensurando especificado foi a concentração de cálcio (\%). Na segunda etapa, foram identificadas as principais fontes de incerteza, utilizando-se um diagrama de causa efeito. Para a massa da amostra, as componentes de incerteza foram: a repetitividade na pesagem; a incerteza na calibração da balança; a estabilidade com o tempo; o efeito da 
temperatura; a linearidade, e a resolução da balança. Para o volume gasto na titulação da amostra, as componentes de incerteza foram: a repetitividade na titulação; a incerteza da calibração da bureta; o efeito do menisco; o efeito da temperatura, e a resolução da bureta. No volume da diluição, as componentes de incerteza foram: a repetitividade; a incerteza na calibração do balão volumétrico; o efeito do menisco, e o efeito da temperatura. As componentes relacionadas com o volume da alíquota foram: a repetitividade na transferência do volume da pipeta; a incerteza na calibração da pipeta; o efeito temperatura, e o efeito do menisco. As componentes relacionadas com a concentração da solução de permanganato de potássio foram: as incertezas da massa de permanganato de potássio, as incertezas da massa molar do permanganato de potássio e o volume da diluição.

Na terceira etapa, foi realizada a quantificação dos componentes de incerteza. Os resultados das incertezas expandidas em um nível de confiança de $95 \%$ foram: $\pm 0,0005 \mathrm{~g}$ com $\mathrm{k}=2,20$ para massa da amostra; $\pm 0,1884 \mathrm{~mL}$ com $\mathrm{k}=2,52$ para o volume gasto na titulação da amostra; $\pm 0,0474 \mathrm{~mL}$ com $\mathrm{k}=2,00$ para a diluição da solução estoque; $\pm 0,0131 \mathrm{~mL}$ com $\mathrm{k}=2,00$ para a alíquota e $\pm 0,0041$ mol. $\mathrm{L}^{-1} \mathrm{com} \mathrm{k}=2,37$ para a concentração da solução de permanganato de potássio. O resultado final da incerteza de medição para o mensurando $\left(\% \mathrm{Ca}^{++}\right)$foi de $\pm 0,00904 \mathrm{~g}$, com um nível de confiança de aproximadamente $95 \%$ e $\mathrm{k}=2,87$. O resultado corrigido da análise de cálcio em salsichas foi de 0,108 $\pm 0,009 \mathrm{~g}$ de cálcio/100 g da amostra.

\section{Conclusões}

A metodologia analítica proposta para quantificação de cálcio, utilizando-se papel filtro quantitativo para a recuperação do precipitado de oxalato de cálcio, mostrou-se sensível, especifica, precisa, exata, robusta e linear na faixa de trabalho, sendo adequada para determinar a concentração de cálcio em produtos cárneos.

\section{Referências bibliográficas}

ABNT - ASSOCIAÇÃO BRASILEIRA DE NORMAS TÉCNICAS. NBR ISO/IEC 17025: requisitos gerais para competência de laboratórios de ensaio e calibração. Rio de Janeiro, 2001. 20 p.

BRASIL. MINISTÉRIO DA AGRICULTURA PECUÁRIA E ABASTECIMENTO. Instrução Normativa n 20, de 21 de julho de 1999. Oficializa os métodos analíticos físico-químicos para controle de produtos cárneos e seus ingredientes - sal e salmoura. Diário Oficial da União, Brasília, 27 de agosto de 1999.

AGÊNCIA NACIONAL DE VIGILÂNCIA SANITÁRIA.

Resolução no 899, de 29 de maio de 2003. Guia para validação de métodos analíticos e bioanalíticos. Diário Oficial da União, Brasília, 02 de junho de 2003.

D 'ACRI, V. Trabalho e saúde na indústria têxtil de amianto. São Paulo em Perspectiva, v. 17 n. 2, 2003.
ELLISON, S. L. R.; ROSSLEIN, M.; WILLIANS, A. (Eds.). Guia eurachem/citac: determinando a incerteza na medição analítica. 2 ed. [s.l.]: Sociedade Brasileira de Metrologia, 2002.

EUROPEAN AGENCY FOR THE EVALUATION OF MEDICINAL PRODUCTS. ICH topic Q2B validation of analytical procedures: methodology. Londres, 1996. Disponível em: <http://www.eudra. org/emea.html>. Acesso em: 10 de agosto de 2007.

FEINBERG, M.; RAGUĖNÈS, N. Development and application of a standardized validation procedure for food chemistry laboratories. Analytica Chimica Acta, n. 391, p. 239-252, 1998.

FOOD AND DRUG ADMINISTRATION. Ora Laboratory Procedure. Methods, method verification. Rockville, 2003. Disponível em: $<$ http://web.ora.fda.gov/dfs/policies/manuals/default.html $>$. Acesso em: 09 de agosto de 2007.

GARBELOTTI, M. L.; TORRES, E. A. F. S.; MARSIGLIA, D. A. P. Determination and validation of dietarry fiber in food by the enzymatic gravimetric method. Food Cheemistry, v. 83, p. 469-473, 2003.

GARDA, J.; MACEDO, R. M.; FURLONG, E. B. Determinação de tricotecenos em cerveja e avaliação de incidência no produto comercializado no Rio Grande do Sul. Ciência e Tecnologia de Alimentos, v. 24, n. 4, p. 657-663, 2004.

INMETRO. Orientações sobre validação de métodos de ensaios químicos. Rio de Janeiro, 2003.

MOHAMED, F. A. et al. A validated spectrofluorimetric method for determination of some psychoactive drugs. Journal of pharmaceutical and Biomedical Analyis, v. 39, n. 1-2, p. 139-146, 2005.

NEVADO, J. J. B. et al. Enantiomeric determination, validation and robustness studies of racemic citalopram in pharmaceutical formulations by capillary electrophoresis. Journal of Chromatography A., v. 1072, p. 249-257, 2005.

PINHO, O. et al. Quantification of synthetic phenolic antioxidants in liver pâtés. Food Chemistry, v. 68, n. 1, p. 353-357, 2000.

PLATZER, D. J.; WHITE, B. A. Development and validation of a gradient HPLC method for the determination of clindamycin and related compounds in a novel tablet formulation. Journal of Pharmaceutic and Biomedical Analysis, v. 41, n. 1, p. 84-88, 2006.

RIBANI, M. Validação em métodos cromatográficos e eletroforéticos. Química Nova, v. 27, n. 5, p. 771-780, 2004.

SABLAYROLLES, C. et al. Development and validation of methods for the trace determination of phthalates in sludge and vegetables. Journal of Chromatography A, v. 1072, n. 2, p. 233-242, 2005.

SOUSA, E. A. et al. Aplicação de redes neurais para avaliação do teor de carne mecanicamente separada em salsicha de frango. Ciência e Tecnologia de Alimentos, v. 23, n. 3, p. 307-311, 2003.

TAN, H. S. I.; XU, J.; ZHENG, Y. Cation-exchange high-performance liquid chromatographic assay of piperazine in some pharmaceutical formulations. Journal of chromatography A, v. 2, n. 693, p. 307-314, 1995.

TAVERNIERS, I.; LOOSE, M. D.; BOCKSTAELE, E. V. Trends in quality in the analytical laboratory, II. Analitical method validation and quality assurance. Trends in Analytical Chemistry, v. 23, n. 8, p. 535-550, 2004. 